\title{
Erst das Vertrauen, dann die Technologie
}

\author{
Anna Sax \\ Lic. oec. publ., MHA, Mitglied der Redaktion
}

\begin{abstract}
Eine neue Studie zielt auf die Überbrückung der Schnittstellen zwischen Spitälern und Krankenversicherern. Es zeigt sich, dass technischer Fortschritt allein nicht zu besseren Lösungen führt. Es braucht Menschen, die miteinander kommunizieren und sich gegenseitig vertrauen.
\end{abstract}

Nichts weniger als die Verbindung zweier Welten wollen die Autoren der Studie bewerkstelligen, die sie im Januar in Zürich präsentierten. Bei den zwei Welten handelt es sich um die Spitalwelt und die Krankenversicherungswelt, zwischen denen sich gemäss Ingo Muschick von der Consultingfirma Synpulse eine «Mauer des Misstrauens» erhebt. Diese gelte es zu überwinden, forderte Alfred Angerer von der Zürcher Hochschule für Angewandte Wissenschaften ZHAW in seinem Einführungsreferat. Als Ort für die Präsentation ihrer Studie ${ }^{1}$ wählten die Organisatoren von Synpulse und ZHAW passend zum Thema eine Brücke. Der «Impact Hub» unter dem Bahnviadukt zwischen Zürich-Wipkingen und dem Hauptbahnhof bildete die urbane Kulisse für eine Begegnung zwischen Spitalund Versicherungswelt, die jedoch wenig Anlass gab zu Optimismus im Hinblick auf die Überwindung von Vorurteilen zwischen diesen beiden Akteuren des Gesundheitswesens. Durch den Event leitete Tagesschau-Moderator Florian Inhauser.

\section{Die Health-Value-Chain}

Schnittstellen gibt es mehr als genug, und Reibungen sind an der Tagesordnung, wie sich bei den Befragungen der Akteure im Rahmen der vorgestellten Studie zeigte. Besonders die Verantwortlichen in den Spitälern scheinen unzufrieden zu sein mit der Qualität der Zusammenarbeit mit den Versicherern. Noch sind wir weit entfernt von der «Health-Value-Chain», wie sie vor 10 Jahren von Porter und Teissberg ${ }^{2}$ empfohlen wurde: Bei einer «Value Chain» bzw. «Wertkette» handelt es sich um ein Konzept, welches den Wertschöpfungsprozess bei der Herstellung und Vermarktung von Produkten darstellt. Übertragen auf den Gesundheitssektor wäre dies die Betrachtung der gesamten Behandlungskette, nicht nur der einzelnen Kostenträ-
D'abord la confiance, puis la technologie

Une étude présentée récemment lors d'un congrès s'est intéressée à la collaboration entre les hôpitaux et les assureurs-maladie. Si les auteurs évoquent un «mur de méfiance» entre les deux branches, des sondages montrent que les hôpitaux jugent la collaboration avec les assureurs nettement moins bonne qu'inversement. Les hôpitaux déplorent en particulier le manque de compréhension des assureurs pour leurs processus, alors que les assureurs aimeraient être davantage intégrés dans les décisions de traitement. Derrière les projets en commun, on retrouve souvent des personnes qui se connaissent déjà. Conclusion des auteurs: sans confiance, aucun partenariat fiable n'est possible. A l'occasion de ce même congrès, il s'est également avéré que les hôpitaux étaient plutôt réticents à investir dans les projets informatiques. Tant que I'on ne sait pas comment va évoluer la cybersanté, et particulièrement le dossier électronique du patient, ils préfèrent investir dans les infrastructures médicales. Enfin, alors que la gestion des données des patients/clients constitue l'activité clé des assureurs, les hôpitaux souhaiteraient réduire les tâches administratives pour pouvoir se concentrer sur leurs patients.

ger und Leistungserbringer: «Das Health-Value-ChainKonzept zielt darauf ab, möglichst nahtlose Übergänge zwischen den Akteuren zu schaffen.» In der Realität führt allerdings nicht nur unternehmerische Vernunft dazu, dass sich zwei Organisationen aufeinander zubewegen, sondern Zufall. Es beginnt zum Beispiel mit zwei Personen, die sich kennen. Daraus ergibt sich vielleicht ein gemeinsames Projekt und schliesslich eine 


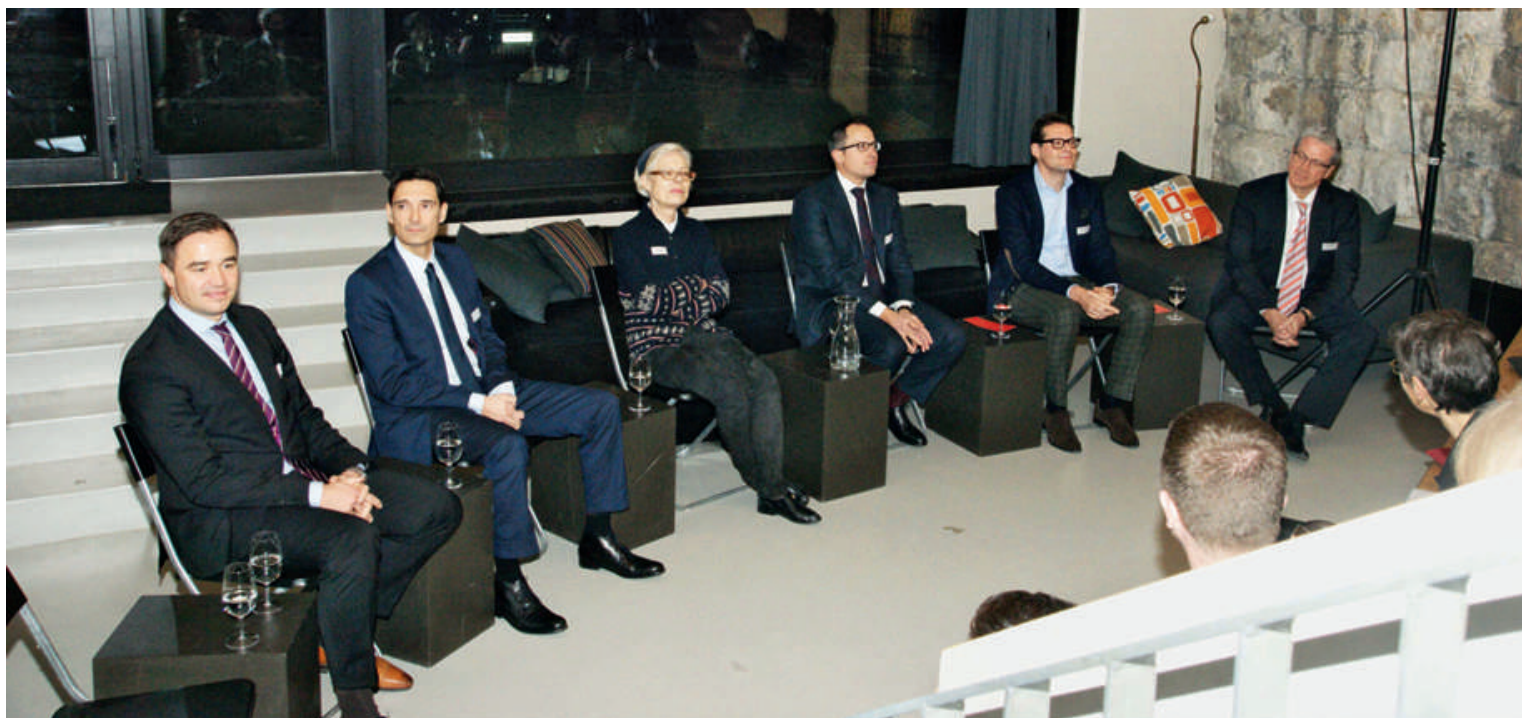

Die «Mauer des Misstrauens» liefert Diskussionsstoff zwischen der Spitaldirektorin Rita Ziegler (3. von links) und Sanjay Singh, Leiter Leistungen bei Sanitas (2. von links).

Bild: $z V g$ von Synpulse

Lösung für die dauerhafte Überwindung einer Schnittstelle. Integration kann also gelingen, muss aber nicht. Sie hängt von persönlichen Beziehungen ab und von der Bereitschaft, Verantwortung für die ganze Behandlungskette zu übernehmen. Oft scheitere sie daran, so beantwortete Angerer eine Frage des Moderators, dass man mit sich selbst beschäftigt sei. Für Beziehungspflege, den Blick über den eigenen Tellerrand, nehme man sich zu wenig Zeit. Sanjay Singh, Leiter Leistungen und Kundenberater bei Sanitas, bemerkte später während der Podiumsdiskussion, er stelle gerne Leute ein, die zuvor im Spital gearbeitet hätten, um die Sensibilität für die Vertragspartner zu fördern. Er selbst war früher in einem Hirslanden-Spital tätig.

\section{Das KWD-Modell}

Wie könnte nun eine Kooperation zwischen Spital und Krankenversicherer konkret aussehen? Singh führte als Beispiel unter anderen eine neue Form der Zusammenarbeit zwischen seiner Versicherung Sanitas und dem Kantonsspital Winterthur an. Dabei geht es um ein Controlling-System mit Zielvorgaben, welches das Erstellen von 31000 ambulanten und 6000 stationären Rechnungen überflüssig mache, so Singh. Generell setzten die Referenten grosse Hoffnungen in eHealthLösungen, allen voran Renato Gunc von der Post, die ihren Service für den Transport von Gesundheitsdaten vorantreiben will. Doch zurück zur Synpulse/ZHAWStudie: Diese beurteilt die Bereitschaft der Akteure, sich in die "Health-Value-Chain» zu integrieren. Das Modell dazu nennt sich «KWD», KWD steht für Können, Wollen, Dürfen. Die Expertenbefragungen zielten also darauf ab zu ermitteln, wie gross das Wissen über die Prozesse an den Schnittstellen ist (Können), wie es mit der Bereitschaft steht, sich als Kettenglied in der «Health-Value-Chain» zu sehen (Wollen), und was das Management tut, um diese Bereitschaft zu fördern (Dürfen). Es zeigte sich, dass zwar die Kooperationsbereitschaft bzw. das «Wollen» vorhanden ist, «Können» und «Dürfen» jedoch noch ausbaufähig sind. Die Spitäler bemängeln insbesondere, dass bei den Versicherern zu wenig Verständnis für die Abläufe und Anforderungen der Leistungserbringung vorhanden sei. Die Versicherer ihrerseits fühlen sich von den Spitälern ungenügend in die Entscheidungen über Behandlungen einbezogen. Generell schätzen die Spitäler die Qualität der Zusammenarbeit mit den Versicherern schlechter ein als umgekehrt. «Warum ist das so? Sind die Spitäler ehrlicher und realistischer?», wollte Moderator Inhauser von den Studienautoren Florian Liberatore (ZHAW) und Christian Ruhse (Synpulse) wissen. «Im Spital sind die Hürden höher», antwortete Liberatore. "Für die Krankenversicherer gehört IT ohnehin zum Kerngeschäft», präzisierte Ruhse. Dass die Spitäler ein anderes Kerngeschäft haben, als sich um IT-Lösungen zu bemühen, zeigte sich in der anschliessenden Diskussion dann deutlich.

Als Moderator Inhauser wissen wollte, wer von Verbesserungen an den Schnittstellen profitiere, erhielt er zur Antwort, dass es weniger um Versorgungsqualität als um administrative Prozesse gehe. Profiteure seien also in erster Linie Krankenversicherer und Spitäler. Der Patient profitiere indirekt. Zwar betonen die Studienautoren, dass sich die "Health-Value-Chain" selbstverständlich um die Patientin herum zu positionieren habe, und stellen dies auch in den Grafiken so dar. Dennoch werden mögliche Vorteile für die Patien- 
ten nur in einem kurzen Abschnitt erläutert. Noch scheint der sogenannte «Perspektivenwechsel» nicht wirklich stattgefunden zu haben.

\section{Pragmatismus und Vertrauen}

Handlungsempfehlungen dürfen nicht fehlen am Ende einer Studie, und so wird wieder das Bild der Brücke präsentiert, deren Fundament die «strategische Positionierung der Akteure» bildet. Darauf aufbauend folgt zunächst die Ausgestaltung der Partnerschaft: «Vertrauen und gemeinsame Ziele sind der Schlüssel zu einer erfolgreichen Partnerschaft! Hierfür sind eine klare Positionierung der Akteure und die entsprechende Ausgestaltung der Partnerschaft essentiell.» Jetzt erst sollen die Betriebe darangehen, ihre Organisationen, Prozesse und IT-Infrastruktur anzupassen. Die Studienautoren plädieren für Pragmatismus, für kontinuierliche Verbesserungen und vor allem: für mehr Vertrauen.

«Frau Ziegler, vertrauen Sie Herrn Singh?», fragte also Herr Inhauser zu Beginn des Podiumsgesprächs die Direktorin des Zürcher Universitätsspitals USZ Rita Ziegler. «Ja», antwortete diese, "aber das Vertrauen ist labil.» Was sie störe, sei die Häufigkeit der Anfragen, seien widersprüchliche Botschaften im Sinne von «Ihr seid zu teuer, und das hätten wir auch noch gerne». Weiter ärgerte sich Ziegler über Kostengutsprachen, die zu spät eintreffen würden. «Wir brauchen sie innert Stunden, nicht nach ein paar Tagen!» Er vertraue seinerseits Frau Ziegler, meinte Sanjay Singh. «Wir haben zwar gemeinsame Projekte mit verschiedenen Kliniken des USZ, doch auf Mitarbeiter-Ebene gibt es Reibereien.» Klar müsse man daran arbeiten, dass die Kostengutsprachen schneller erfolgen können. "Aber das dauert.» Und so zog sich die Diskussion weiter. Immer mehr kristallisierte sich heraus, dass die Spitäler kaum bereit sein werden, Geld in IT-Projekte zu investieren, deren Nutzen noch nicht absehbar ist. Mehrfach betonte Ziegler, dass ihre Prioritäten woanders liegen würden, nämlich bei einer guten Medizin. «Unser Kerngeschäft ist die Medizin, alles andere ist Drumherum.»

\section{Kurzinterview mit Beat Moll, Vorsitzender der Geschäftsleitung, Seeklinik Brunnen}

Schweizerische Ärztezeitung: Herr Moll, Sie waren bis vor einem Jahr Mitglied der Geschäftsleitung einer Krankenversicherung. Heute sind Sie CEO eines Spitals. Sie wären also prädestiniert als Botschafter für mehr Kooperation zwischen Krankenversicherern und Spitälern.

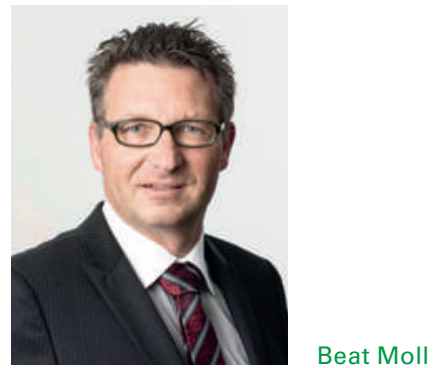

Beat Moll: Ich kann tatsächlich beide Sichtweisen verstehen. Der Wechsel in die Leitung eines Spitals hat mich aber ein Stück weit ernüchtert, was die rasche Weiterentwicklung von eHealth betrifft.

\section{Weshalb die Ernüchterung?}

Das Kerngeschäft der Versicherer ist das Handling von Kundendaten. Die Spitäler haben andere Prioritäten. Wir kümmern uns in erster Linie um das Wohl der Patientinnen und Patienten. IT-Lösungen stehen dabei nicht im Vordergrund.

Aber Sie könnten sich die Arbeit erleichtern, wenn Sie die Kooperation mit den anderen Playern im Gesundheitswesen pflegen würden.

Wir kooperieren selbstverständlich mit unseren Partnern, seien es andere Leistungserbringer, Behörden oder Versicherer. Das gehört zu einer guten Patientenbetreuung. Für grosse IT-Projekte haben wir aber im Moment die Ressourcen nicht zur Verfügung.

\section{Also machen die Spitäler nicht mit bei eHealth?}

In der Schweiz gibt es im Moment noch einen Flickenteppich von verschiedensten eHealth-Projekten. Und solange wir nicht wissen, wie es mit dem elektronischen Patientendossier weitergeht, werden wir bestimmt keine grossen Investitionen tätigen. Selbstverständlich haben auch wir unsere kleinen eHealthLösungen - doch das sind eher einfache und isolierte Anwendungen.

Die Patientinnen könnten doch von einer durchgehenden "Health-Value-Chain" profitieren, indem sie weniger Wartezeiten und Doppelspurigkeiten in Kauf nehmen müssten?

Die Generation der «Babyboomer» legt grossen Wert auf Datenschutz, mehr als die nachfolgenden Generationen. IT-Lösungen für die Überbrückung der Schnittstellen zwischen den Versicherern und den Leistungserbringern sind also nicht in jedem Fall im Interesse der Patientinnen bzw. der Versicherten. Das kann sich natürlich ändern, wenn die offenen Fragen zum Datenschutz, zu den technischen Rahmenbedingungen und zur Finanzierung geklärt sind. 